\title{
Test-retest reliability of lower limb isokinetic endurance in COPD: a comparison of angular velocities
}

This article was published in the following Dove Press journal:

International Journal of COPD

18 June 2015

Number of times this article has been viewed

\section{Fernanda Ribeiro* \\ Pierre-Alexis Lépine* \\ Corine Garceau-Bolduc \\ Valérie Coats \\ Étienne Allard \\ François Maltais \\ Didier Saey}

Centre de recherche de l'Institut Universitaire de cardiologie et de pneumologie de Québec, Université Laval, Québec, Canada

*These authors contributed equally to this work
Correspondence: Didier Saey Institut Universitaire de cardiologie et de pneumologie de Québec, 2725 Chemin Ste-Foy, Québec, Canada GIV 4G5

Tel + l $41865626 \mid 4$

$\mathrm{Fax}+\mathrm{I} 4186564762$

Email didier.saey@rea.ulaval.ca
Background: The purpose of this study was to determine and compare the test-retest reliability of quadriceps isokinetic endurance testing at two knee angular velocities in patients with chronic obstructive pulmonary disease (COPD).

Methods: After one familiarization session, 14 patients with moderate to severe COPD (mean age $65 \pm 4$ years; forced expiratory volume in 1 second $\left(\mathrm{FEV}_{1}\right) 55 \% \pm 18 \%$ predicted) performed two quadriceps isokinetic endurance tests on two separate occasions within a 5-7-day interval. Quadriceps isokinetic endurance tests consisted of 30 maximal knee extensions at angular velocities of $90^{\circ}$ and $180^{\circ}$ per second, performed in random order. Test-retest reliability was assessed for peak torque, muscle endurance, work slope, work fatigue index, and changes in $\mathrm{FEV}_{1}$ for dyspnea and leg fatigue from rest to the end of the test. The intraclass correlation coefficient, minimal detectable change, and limits of agreement were calculated.

Results: High test-retest reliability was identified for peak torque and muscle total work at both velocities. Work fatigue index was considered reliable at $90^{\circ}$ per second but not at $180^{\circ}$ per second. A lower reliability was identified for dyspnea and leg fatigue scores at both angular velocities.

Conclusion: Despite a limited sample size, our findings support the use of a 30-maximal repetition isokinetic muscle testing procedure at angular velocities of $90^{\circ}$ and $180^{\circ}$ per second in patients with moderate to severe COPD. Endurance measurement (total isokinetic work) at $90^{\circ}$ per second was highly reliable, with a minimal detectable change at the $95 \%$ confidence level of $10 \%$. Peak torque and fatigue index could also be assessed reliably at $90^{\circ}$ per second. Evaluation of dyspnea and leg fatigue using the modified Borg scale of perceived exertion was poorly reliable and its clinical usefulness is questionable. These results should be useful in the design and interpretation of future interventions aimed at improving muscle endurance in COPD.

Keywords: chronic obstructive pulmonary disease, lower limb, isokinetic endurance, testretest reliability

\section{Introduction}

Limb muscle dysfunction has important clinical and prognostic consequences in chronic obstructive pulmonary disease (COPD). In fact, reduced mass and weakness of the quadriceps have been associated with reduced survival in this disease.$^{1-3}$ Muscle strength is the most commonly assessed skeletal muscle function in COPD. However, muscle strength represents only one aspect of muscle function, mostly reflecting the action of fast-twitch muscle fibers. Muscle endurance is defined as the ability to maintain a given task over a certain period of time, and it is determined by muscle fiber oxidative capacity, and the oxygen supply. ${ }^{4}$ In COPD, muscle endurance is impaired to a greater extent than strength. ${ }^{5,6}$ Impaired muscle endurance may even be present in 
patients with mild disease and preserved physical activity, ${ }^{7,8}$ and cannot be predicted from strength. ${ }^{7}$

Despite its relevance to activities of daily living such as walking, ${ }^{9,10}$ muscle endurance is generally not an outcome in COPD clinical trials. ${ }^{11}$ One important methodological issue with the measurement of muscle endurance is the fact that there is no standardization or agreement on a testing protocol that could be used in clinical practice or research. It can be determined with various methodologies, amongst which isokinetic testing is one of the most reliable methods. ${ }^{12}$ The main advantage of this method is that it provides a dynamic measurement of muscle torque while controlling for angular velocities, amplitude, and duration of movement in such a way that the testing conditions could be easily reproduced in subsequent testing. ${ }^{13}$

As with any other muscle function assessment, isokinetic endurance is required to be accurate and reliable. The reliability of isokinetic testing has been reported for strength and endurance in healthy subjects. ${ }^{14,15}$ In COPD patients, although the reliability of isokinetic strength testing has been reported, ${ }^{16}$ little is known about the reliability of isokinetic endurance measurements at different angular velocities.

Based on the model developed by Walter et $\mathrm{al}^{17}$ and the clinical decision-making process inherent in exercise testing protocols described by Charter et al, ${ }^{18}$ we hypothesize that, for both velocities, muscle endurance and work fatigue indices would achieve a minimally acceptable value, defined by an intraclass coefficient (ICC) $>0.6 .{ }^{17}$

The purpose of this study was to determine the test-retest reliability and minimal detectable change (MDC) of 30-maximum repetition isokinetic endurance testing of the quadriceps performed at $90^{\circ}$ and $180^{\circ}$ per second regarding peak torque, muscle endurance, and fatigue in patients with COPD.

\section{Materials and methods}

\section{Subject characteristics}

Patients with stable and moderate to severe stage COPD accordingly to the Global Initiative for Chronic Obstructive
Lung Disease ${ }^{19}$ were recruited. All subjects were former smokers, physically inactive, and naïve to the isokinetic procedure. Exclusion criteria were: other medical conditions that could affect exercise testing (ie, neuromuscular and/or orthopedic disorders, recent cancer, unstable cardiac disease, type 2 diabetes, asthma), recent COPD exacerbations ( $<6$ weeks), body mass index $>35 \mathrm{~kg} / \mathrm{m}^{2}$, rest hypoxemia $\left(\mathrm{SpO}_{2}<90 \%\right)$ or oxygen therapy, regular practice of physical activity (Voorrips score $>9$ ), ${ }^{20}$ and involvement in a rehabilitation or structured exercise training program during the previous 6 months. The research protocol was approved by the ethics committee of the Institut Universitaire de cardiologie et de pneumologie de Québec. All the subjects signed the informed consent before enrolment in the study.

\section{Study design}

Study participation involved three visits. The first visit included a review of medical history, a Voorrips physical activity questionnaire, anthropometric measurements, pulmonary function testing, and a familiarization session for the quadriceps isokinetic endurance test. During familiarization, subjects were asked to perform warm-up and endurance exercise measurements on the isokinetic dynamometer in the same fashion as required for the testing days (described below). The procedures and series of exercises were repeated as needed until performance was considered compatible with acceptable comprehension of the test. ${ }^{14,21}$ After 5-7 days, subjects returned to the laboratory for the quadriceps isokinetic endurance testing protocol, which was repeated at a third visit performed 5-7 days later, as detailed in Figure 1.

The randomization plan was provided by software available online using the number of subjects to be included in the study $(n=14)$ and the number of tests $(n=2)$ performed.

\section{Physical activity}

In order to include subjects with a sedentary lifestyle, the level of physical activity in daily living was assessed with the
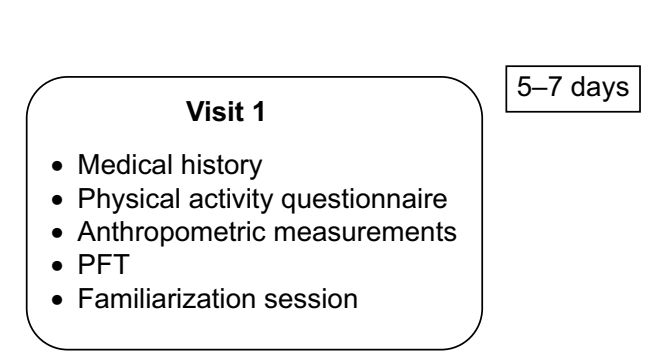

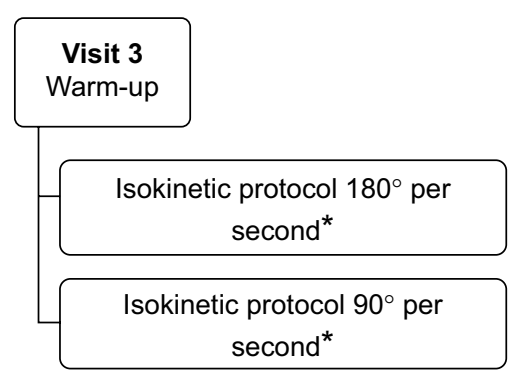

Figure I Experimental design.

Note: *Randomized order.

Abbreviation: PFT, pulmonary function tests.

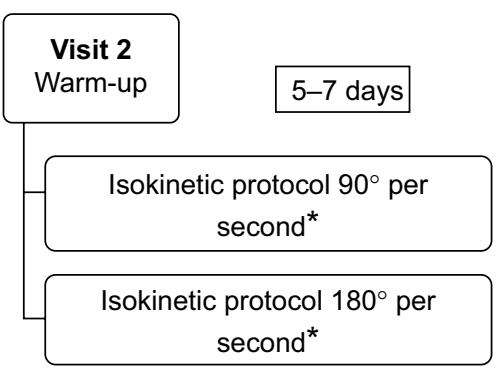


activity questionnaire firstly devised by Baecke et $\mathrm{al}^{22}$ adapted for elderly subjects by Voorrips et $\mathrm{al}^{20}$ and used for patients with COPD. ${ }^{6}$ Subjects were considered sedentary whenever the global Voorrips score, which includes household, sport, and other leisure time physical activity, was lower than 9. None of the patients were enrolled in rehabilitation, exercise training, or physical activity programs during the 6 months prior to inclusion.

\section{Anthropometric data and body composition}

Body composition was assessed by bioelectrical impedance analysis (TBF-300WA Tanita, Arlington Heights, IL, USA), which provides data on fat mass and fat-free mass. Fat-free mass index (FFMI) was calculated as FFMI $=$ FFM/height ${ }^{2}{ }^{23}$

\section{Pulmonary function testing}

Standard pulmonary function tests, including post-bronchodilator spirometry, lung volumes, and carbon monoxide diffusion capacity, were obtained in all subjects during the first visit according to previously described guidelines ${ }^{24-26}$ and related to predicted normal values. ${ }^{27}$

\section{Assessment of quadriceps isokinetic endurance}

All tests of the dominant knee extensors (quadriceps muscle action) were performed using an isokinetic dynamometer (System Pro 4, Biodex Medical Systems, Shirley, New York, NY, USA) and were conducted and recorded by the same investigator (FR). Subjects were positioned and stabilized on the dynamometer chair, as previously described. ${ }^{14,28}$ The knee joint center was aligned to the dynamometer axe center. The lever arm of the dynamometer was firmly attached $3 \mathrm{~cm}$ above the lateral malleolus. Total knee joint displacement was fixed between $90^{\circ}$ and $10^{\circ}$ of knee flexion for all tests. Reference points for chair and lever arm positioning were annotated in order to ensure the same positioning during subsequent visits. As a warm-up procedure, the subjects performed one series of ten submaximal knee extensions. They were then instructed and verbally encouraged to perform 30 maximal knee extensions consecutively and throughout the preset full range of movement. ${ }^{28}$ Subjects were instructed to avoid any muscle effort during return of the leg to the resting position. They performed two trials in a randomized order, one at each angular velocity, ie, $90^{\circ}$ and $180^{\circ}$ per second, each separated by a 1 -hour resting period.

Data for the first contraction were systematically excluded from all analyses, since the starting maneuver is often submaximal. Peak torque was determined from the highest torque measured in 30 contractions. Isokinetic muscle endurance was defined as total muscle work developed in all contractions. Muscle fatigue was assessed using both the fatigue index and the work slope. The fatigue index was determined from the ratio of the work performed during the last ten repetitions to the work performed during the first ten repetitions, ${ }^{14}$ and the work slope was quantified from the decline in muscle work over time throughout the 30 repetitions. ${ }^{21}$

\section{Effort perception}

Perception of dyspnea and leg fatigue was measured using the modified Borg scale (0-10) of perceived exertion ${ }^{29}$ and is reported as the change in scores before and immediately after completion of each isokinetic endurance test ( $\Delta$ dyspnea and $\Delta$ leg fatigue).

\section{Statistical analysis}

Descriptive statistics are reported for subject characteristics and for isokinetic total work, isokinetic work slope, muscle fatigue index, $\Delta$ dyspnea, and $\Delta$ leg fatigue at each visit. The data are expressed as the mean \pm standard deviation. For each subject, comparisons between two isokinetic endurance tests at two angular velocities were performed using a mixed linear model with a hierarchical structure. Results were considered to be statistically significant when the $P$-value was $\leq 0.05$. Test-retest reliability was assessed with $\mathrm{ICC}^{30}$ for peak torque, muscle endurance (total work), rate of decline in muscle work (work slope), fatigue index, $\Delta$ dyspnea, and $\Delta$ leg fatigue, and for each velocity separately. In order to facilitate clinical interpretation of the reliability results, MDC values at the $95 \%$ confidence level, both in absolute $\left(\mathrm{MDC}_{95 \%}\right)$ and relative $\left(\mathrm{MDC}_{95 \%}[\%]\right)$ terms, were calculated from the standard error of measurement (SEM) using the following formulae described by Beckerman et al: ${ }^{31}$

$$
\begin{gathered}
\mathrm{MDC}_{95 \%}=\mathrm{SEM} \times 1.96 \times \sqrt{2} \\
\mathrm{MDC}_{95 \%}(\%)=\frac{\mathrm{MDC}_{95 \%}}{\text { Mean of } 2 \text { visits }} \times 100
\end{gathered}
$$

Finally, between-measurement limits of agreement were established using a Bland and Altman ${ }^{32}$ representation for both angular velocities. The proportion of scores at two standard deviations of the mean difference between test-retest values was taken as a parameter of agreement.

The statistical analyses were performed using the statistical packages $\mathrm{R}$ version 3.0.2. (R Foundation for Statistical Computing, Vienna, Austria.), SAS version 9.4 (SAS 
Table I Subject characteristics $(n=\mid 4)$

\begin{tabular}{|c|c|}
\hline Male/female & $11 / 3$ \\
\hline Age (years) & $65 \pm 4$ \\
\hline Body mass index $\left(\mathrm{kg} / \mathrm{m}^{2}\right)$ & $26.6 \pm 3.9$ \\
\hline Fat-free mass index $\left(\mathrm{kg} / \mathrm{m}^{2}\right)$ & $18.6 \pm 1.7$ \\
\hline FVC (L) & $3.6 \pm 1.1$ \\
\hline FVC (\% predicted) & $97 \pm 25$ \\
\hline $\mathrm{FEV}_{1}(\mathrm{~L})$ & $1.51 \pm 0.59$ \\
\hline $\mathrm{FEV}_{1}$ (\% predicted) & $55 \pm 18$ \\
\hline $\mathrm{FEV}_{\mathrm{I}} / \mathrm{FVC}(\%)$ & $42 \pm 10$ \\
\hline TLC (L) & $7.1 \pm 1.8$ \\
\hline TLC (\% predicted) & $117 \pm 10$ \\
\hline $\mathrm{DL}_{\mathrm{co}}(\%$ predicted $)$ & $64 \pm 16$ \\
\hline $\mathrm{SpO}_{2}(\%)$ & $96 \pm 1$ \\
\hline Physical activity (Voorrips score) & $5 \pm 5$ \\
\hline
\end{tabular}

Note: Values are shown as the mean \pm standard deviation.

Abbreviations: FVC, forced vital capacity; $\mathrm{FEV}_{1}$, forced expiratory volume in I second; TLC, total lung capacity; $\mathrm{DL}_{\mathrm{co}}$, diffusing capacity of the lung for carbon monoxide; $\mathrm{SpO}_{2}$, pulse oximetry oxygen saturation.

Institute Inc, Cary, NC, USA), Statistical Package for the Social Sciences version 22 software (IBM Corporation, Armonk, NY, USA) or GraphPad Prism version 6 for Mac OS X (GraphPad Software, San Diego, CA, USA, www. graphpad.com).

\section{Results}

Subject characteristics are presented in Table 1. Fourteen sedentary patients with COPD (78\% men) completed the study. Except for one subject for whom an additional trial at $180^{\circ}$ per second was necessary, all participants required only one practice session for familiarization with the isokinetic testing procedure. In agreement with randomization, eight participants began with the endurance test performed at $180^{\circ}$ per second and six began at $90^{\circ}$ per second for the first visit, and the order of test administration was reversed for the second visit. Peak torque, total isokinetic work, muscle fatigue, and delta rating of dyspnea and leg fatigue for each endurance trial and visit at both velocities $\left(90^{\circ}\right.$ and $180^{\circ}$ per second) are shown in Table 2 . As expected, statistically significant differences in these variables were noted between the two angular velocities $(P<0.05)$.

Table 3 shows the ICC and MDC for the two quadriceps endurance testing protocols. At $90^{\circ}$ per second, an ICC $>0.90$ was found for peak torque, total isokinetic work, work slope, and work fatigue index. In contrast, at $180^{\circ}$ per second, peak torque and total isokinetic work were the only parameters with an ICC $>0.75$. The $\mathrm{MDC}_{95 \%}(\%)$ for peak force at $90^{\circ}$ and $180^{\circ}$ per second were $12 \%$ and $19 \%$, respectively. The $\mathrm{MDC}_{95 \%}(\%)$ for total isokinetic work measurements at $90^{\circ}$ and $180^{\circ}$ per second was $10 \%$ and $19 \%$, respectively. The $\mathrm{MDC}_{95 \%}(\%)$ for work slope showed poor reliability at both velocities. However, the work fatigue index measurements were considered reliable only at $90^{\circ}$ per second, with a $\mathrm{MDC}_{95 \%}(\%)$ of $13 \%$, in comparison with $85 \%$ at $180^{\circ}$ per second. Measurements of perception of dyspnea and leg fatigue were poorly reliable, with an ICC $<0.65$ and $\mathrm{MDC}_{95 \%}(\%)$ $>70 \%$ irrespective of muscle contraction velocity.

Limits of agreement, by Bland-Altman plots, are presented in Figure 2A-L. Better between-visits reliability, expressed as a percentage of difference (bias \pm random error) was found for muscle endurance at $90^{\circ}$ per second when compared with $180^{\circ}$ per second $(-2.6 \% \pm 10.1 \%$ and $5.4 \% \pm 19.7 \%$, respectively). Similar results were found for work slope and work fatigue index, with 95\% confidence interval limits of agreement much wider at $180^{\circ}$ per second, implying a lower reliability for these measurements at this velocity. Bland-Altman plots for dyspnea and leg fatigue scores clearly illustrate a high between-visits variability in changes.

\section{Discussion}

The major finding of this study was that a 30-maximum repetition isokinetic muscle endurance test can be performed in a reliable fashion in sedentary patients with moderate to severe COPD and similar body composition, particularly at $90^{\circ}$ per second. While isokinetic peak torque and total muscle work

Table 2 Comparison between variables of the lower limb isokinetic endurance test at different angular velocities

\begin{tabular}{|c|c|c|c|c|}
\hline \multirow[t]{2}{*}{ Variables } & \multicolumn{2}{|c|}{$90^{\circ}$ per second } & \multicolumn{2}{|c|}{$180^{\circ}$ per second* } \\
\hline & Visit I & Visit 2 & Visit I & Visit 2 \\
\hline Peak torque (Nm) & $115 \pm 30$ & $118 \pm 33$ & $89 \pm 26$ & $89 \pm 25$ \\
\hline Total isokinetic work (Nm) & $2,164 \pm 553$ & $2,214 \pm 535$ & $1,7 \mid 2 \pm 508$ & $|, 803 \pm 5| 4$ \\
\hline Work slope (\%/rep) & $-2.4 \pm 0.8$ & $-2.5 \pm 0.9$ & $-1.5 \pm 0.7$ & $-1.3 \pm 0.6$ \\
\hline Fatigue index (\%) & $49.3 \pm 8.2$ & $49.5 \pm 7.9$ & $40.1 \pm 13.0$ & $35.4 \pm I 4.7$ \\
\hline Delta dyspnea (Borg scale) & $3 \pm 2$ & $3 \pm 1$ & $2 \pm 1$ & $3 \pm 1$ \\
\hline Delta leg fatigue (Borg scale) & $5 \pm 3$ & $6 \pm 2$ & $4 \pm 2$ & $5 \pm 1$ \\
\hline
\end{tabular}

Notes: Values are shown as the mean \pm standard deviation. $* P<0.05180^{\circ}$ per second vs $90^{\circ}$ per second. 
Table 3 Test-retest reliability

\begin{tabular}{|c|c|c|c|c|c|c|}
\hline \multirow[t]{2}{*}{ Variables } & \multicolumn{3}{|l|}{$90^{\circ}$ per second } & \multicolumn{3}{|l|}{$180^{\circ}$ per second } \\
\hline & $\operatorname{ICC}(95 \% \mathrm{CI})$ & MDC $_{95 \%}$ & $\operatorname{MDC}_{95 \%}(\%)$ & ICC (95\% CI) & MDC $_{95 \%}$ & MDC $_{95 \%}(\%)$ \\
\hline Peak torque & $0.97(0.92-0.99)$ & $14 \mathrm{Nm}$ & 12 & $0.94(0.8 \mathrm{I}-0.98)$ & $17 \mathrm{Nm}$ & 19 \\
\hline Total isokinetic work & $0.98(0.93-0.99)$ & $220 \mathrm{Nm}$ & 10 & $0.93(0.78-0.98)$ & $339 \mathrm{Nm}$ & 19 \\
\hline Work slope & $0.94(0.83-0.98)$ & $0.58 \% /$ rep & 23 & $0.59(0.13-0.85)$ & $1.21 \% / \mathrm{rep}$ & 86 \\
\hline Work fatigue index & $0.92(0.77-0.97)$ & $6.5 \%$ & 13 & $0.30(-0.24-0.70)$ & $32.2 \%$ & 85 \\
\hline$\Delta$ Dyspnea & $0.5 \mathrm{I}(-0.03-0.8 \mathrm{I})$ & 3 & 118 & $0.24(-0.26-0.66)$ & 3 & 121 \\
\hline$\Delta$ Leg fatigue & $0.5 \mathrm{I}(-0.02-0.8 \mathrm{I})$ & 5 & 86 & $0.60(0.15-0.85)$ & 3 & 75 \\
\hline
\end{tabular}

Abbreviations: $\mathrm{Cl}$, confidence level; ICC, intraclass correlation; $\mathrm{MDC}$, minimal detectable change; $\mathrm{MDC}_{95 \%}$, absolute value of $\mathrm{MDC}_{\text {at }}$ the $95 \% \mathrm{Cl} \mathrm{MDC}_{95 \%}(\%)$, relative value of $\mathrm{MDC}$ at the $95 \% \mathrm{Cl}$; rep, repetition.

were highly reliable at angular velocities of $90^{\circ}$ and $180^{\circ}$ per second, test-retest measurements of work slope and fatigue index were only reproducible at $90^{\circ}$ per second.

Changes in dyspnea and leg fatigue perception scores failed to achieve acceptable test-retest reproducibility, irrespective of the velocity applied. Therefore, their assessment in this isokinetic endurance test is of uncertain significance. Overall, our results have practical implications for development of endurance muscle testing in patients with $\mathrm{COPD}$, an area for which no standardization is available.

To our knowledge, this is the first study to provide a MDC and to report on comparison of the reliability of quadriceps muscle endurance indices at two angular velocities commonly used to assess muscle endurance and fatigue in isokinetic protocols in COPD. Typically, in comparison with healthy controls, patients with COPD are found to present lower isometric torque and total work. ${ }^{33,34}$

Results for the ICC analyses, MDC, and Bland-Altman plots all show that a velocity of $90^{\circ}$ per second provides more robust data than a faster velocity of $180^{\circ}$ per second. These findings are consistent with a previous study involving healthy subjects that reported a much lower reliability for muscle fatigue indices in a similar 30 repetitions trial at $180^{\circ}$ per second than at $60^{\circ}$ per second. ${ }^{14}$ The higher reliability for endurance (total isokinetic work) compared with the fatigue index observed in our study is also in line with previous studies in healthy subjects that consistently found the strongest and most reliable measures of isokinetic endurance were total isokinetic work and average power, irrespective of angular velocity. ${ }^{12,14,35,36}$ In those studies, low reliability was found for work slope or fatigue index.

There are several potential explanations for the higher reliability at lower contraction velocity observed in our study. Muscle can produce greater dynamic torque during an isokinetic maneuver when contracting slowly rather than at faster velocities..$^{37,38}$ This could be explained by difficulties in fully activating the available motor units by voluntary effort over the whole range of motion or in the brief time required for full knee extension at faster speeds of contraction. ${ }^{37,38}$ In addition, elderly individuals show significant deficiencies in functional performance ${ }^{39}$ and typically do not produce fast contractions in daily life. This could contribute to a reduction in their torque production and total work using a high velocity protocol. Lastly, despite our effort to minimize the impact of any learning effects with a rigorous familiarization visit, it is still possible that patients may not have become fully accustomed to the high velocity protocol.

From perceptual and symptomatic perspectives, our data show that the variations in dyspnea and leg fatigue scores induced by the isokinetic endurance protocol were not reliable between the two visits at either velocity. Values obtained from the $\mathrm{MDC}_{95 \%}$ regarding variation in dyspnea showed \pm 3 points in Borg scale variability, which represents more than half the range of the scale. Similar results were observed for leg fatigue. The reliability of the Borg ratings of perceived exertion during exercise has already been challenged in numerous publications involving healthy subjects ${ }^{40,41}$ and patients with COPD. ${ }^{42}$ In a study by Mador et a ${ }^{42}$ evaluating six patients with moderate to severe COPD during weekly symptom-limited maximal incremental exercise on a cycle ergometer, it was estimated that an individual subject's maximum Borg score would need to change by $36 \%$ or more before a significant change from baseline could be inferred. On the contrary, in multicenter trials involving 463 COPD patients, O'Donnell et al ${ }^{43}$ demonstrated an ICC of 0.79 (0.76-0.82) for Borg dyspnea ratings at isotime exercise and of $0.81(0.77-0.84)$ at peak exercise during two repeated cycling exercises. To our knowledge, the reliability of the modified Borg dyspnea scale has not been assessed in any endurance isokinetic protocol involving patients with COPD. The reasons for this apparent discrepancy in the reliability of dyspnea rating between isokinetic muscle testing and cycling exercise are unclear. It may well be that the type of exercise, with cycling exercise inducing a larger degree of dyspnea 
A

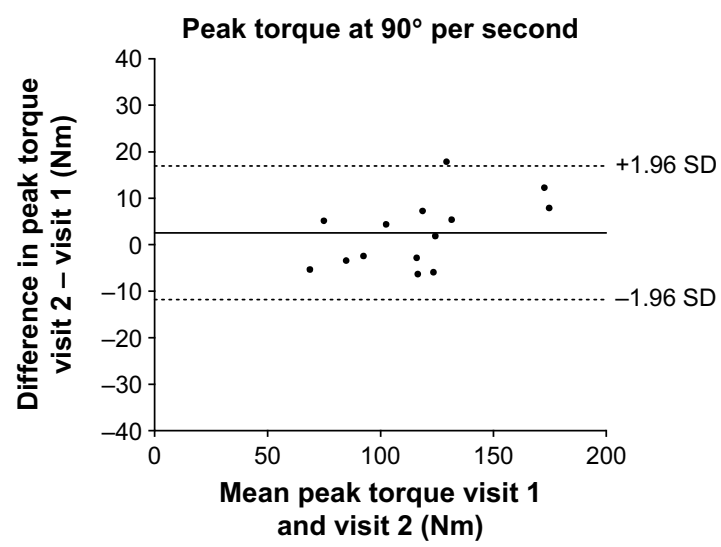

C

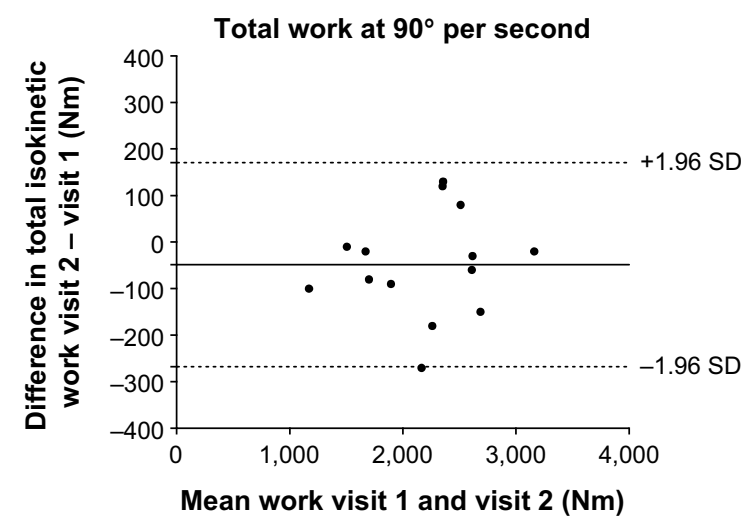

E

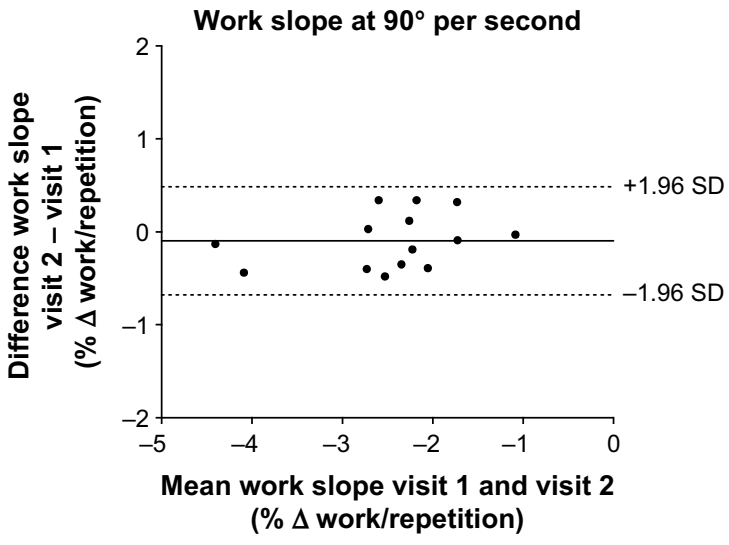

G

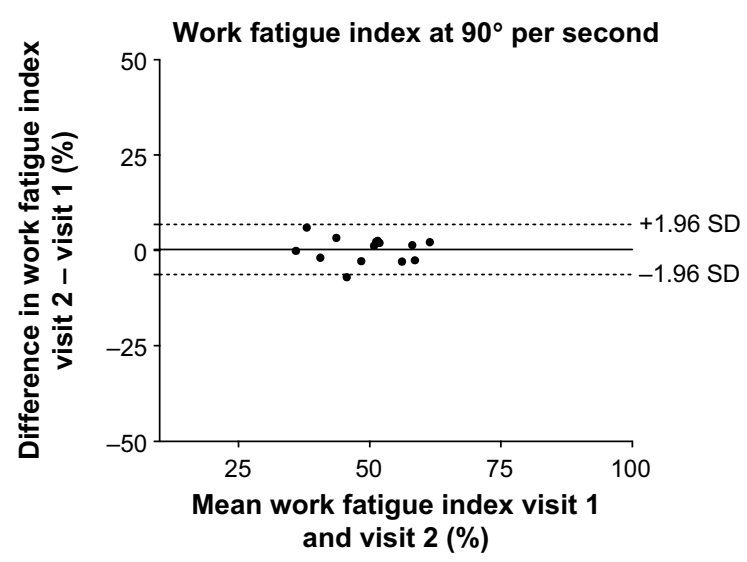

B

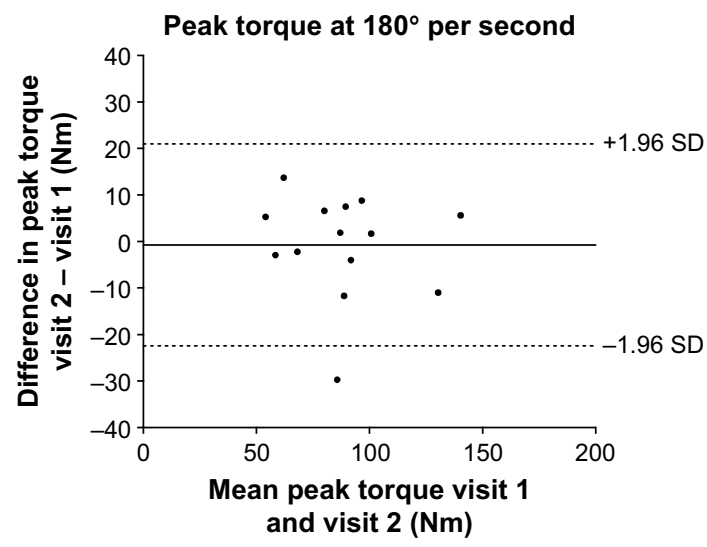

D

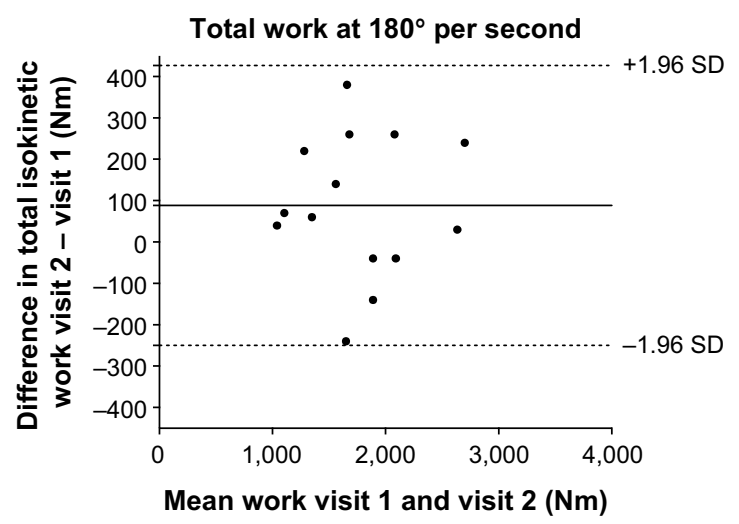

F

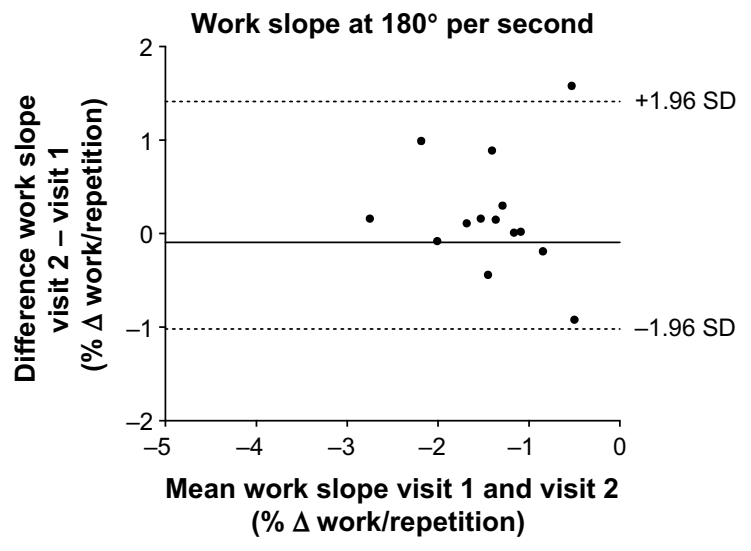

H

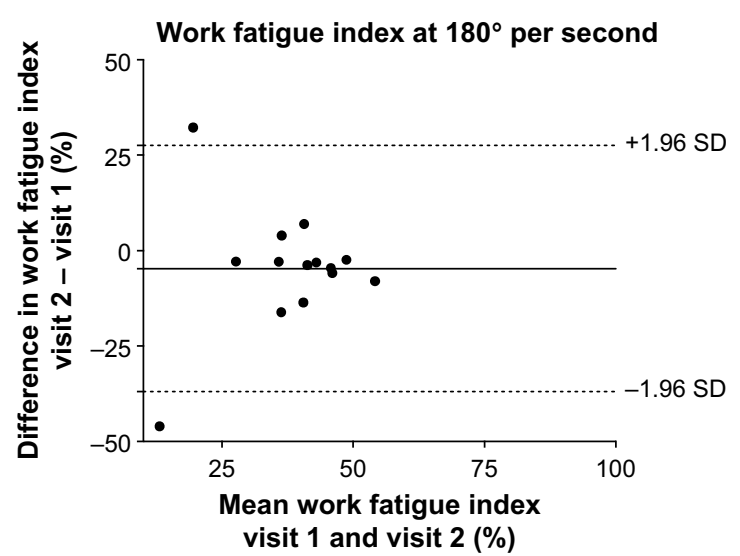

Figure 2 (Continued) 

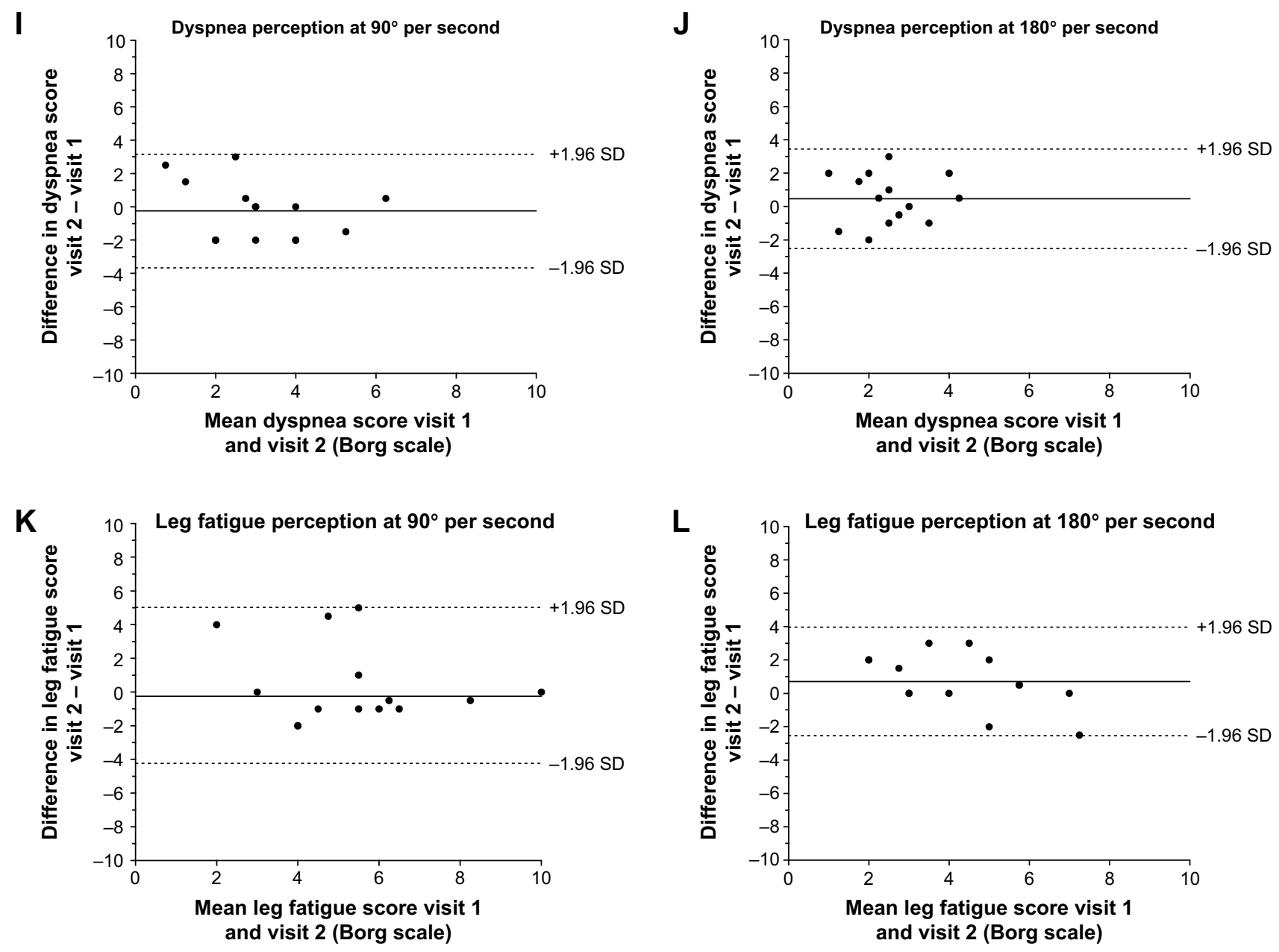

Figure 2 Bland-Altman plots.

Notes: Ilustration by Bland-Altman plots of means differences (solid lines) and $95 \%$ confidence intervals (dotted lines) of a 30-maximal repetition isokinetic muscle endurance procedure performed on two separate occasions for peak torque, total work, work slope, work fatigue, dyspnea perception, and leg fatigue perception. Left panels illustrate means differences and $95 \%$ confidence intervals for peak torque (A), total work (C), work slope (E), work fatigue (G), dyspnea perception (I), and leg fatigue perception (K) during isokinetic leg extension performed at $90 \%$ s. The right panels illustrate means differences and $95 \%$ confidence intervals for peak torque (B), total work (D), work slope $(\mathbf{F})$, work fatigue $(\mathbf{H})$, dyspnea perception $(\mathbf{J})$, and leg fatigue perception $(\mathbf{L})$ during isokinetic leg extension performed at $180^{\circ} / \mathrm{s}$.

Abbreviation: SD, standard deviation.

(typically $5-6$ points on a 10 -point Borg scale) than isokinetic muscle testing ( $2-3$ points) in the present study, exerts much influence on the reliability of this dyspnea scale.

It could be argued that a limitation of our study is the relatively small sample size. However, several factors favor its external validity for a precise population of patients with COPD. Firstly, the sample size was determined to test the hypothesis that, for both velocities, muscle endurance and work fatigue indices would achieve minimally acceptable reliability, such as defined by an ICC $>0.6 .{ }^{17}$ Moreover, increasing the sample size does not necessarily change reliability. A study involving a healthy larger population demonstrated that reliability, measured by Pearson correlation coefficients, may be more affected by similarities between subjects than by the sample size. ${ }^{44}$ This study, including 176 subjects, led to an ICC $>0.7$, which was clinically acceptable. Taking into account the complexity of phenotypes commonly found in COPD, we restricted our study population to a very homogenous sample in terms of severity of disease, body composition, and level of physical activity. Additionally, a separate familiarization session was provided to minimize within-session learning effects. Accordingly to Walter et a ${ }^{17}$ with 14 subjects performing two observations for each velocity, this study is adequately powered to demonstrate the minimally acceptable reliability in a homogenous population.

It would have been of interest to test the reliability of the muscle endurance protocols separately in men and women. Although this could not be done in our study, we found that excluding the three women from the analysis did not alter the conclusions about the reliability of the measurements. Additionally, subjects' baseline muscular condition might have been different between visits, potentially influencing 
our conclusions concerning the reliability of the measurements. However, the close similarity in maximal peak torque measurements on subsequent testing days suggests similar baseline conditions between visits and no impact of the fatigue induced by the 30 maximal contractions in the first set on performance during the second test in the same visit.

Several studies have reported on quadriceps endurance in COPD using a variety of methodologies, including measurement of time to sustain submaximal voluntary contractions at a preset proportion of submaximal voluntary contractions using weights,,${ }^{6,75-47}$ a strain gauge, ${ }^{48}$ or a dynamometer, ${ }^{5}$ quantification of the number of contractions or fatigue index using an isokinetic protocol, ${ }^{49}$ and time taken for quadriceps strength to fall to a certain threshold following repeated magnetic stimulation of the femoral nerve. ${ }^{3}$ Collectively, these studies clearly show that lower limb muscle endurance is reduced in COPD in comparison with healthy controls of similar age and that muscle endurance is compromised to a larger extent than strength. ${ }^{5,7}$ These studies also illustrate the relationship between endurance and oxidative capacity. ${ }^{3,48}$ Altogether, these studies provide a strong rationale for the relevance of measuring muscle endurance in COPD.

Despite the interest in quantifying muscle endurance, this parameter has not been incorporated into clinical practice. In this regard, the diversity of protocols is a reflection of the absence of standardization and highlights a need to develop standardized, simple, but valid and sensitive endurance knee extensor procedures to be used in usual clinical settings in patients with COPD. Also, the lack of consensus with regard to designation of the best testing protocol to evaluate muscle endurance and the absence of predictive values are other impediments to the widespread application of this measurement. The concise protocol that we report here has the advantage of feasibility in patients with COPD in regard to their cardiorespiratory limitation, relative simplicity, and reproducibility, allowing measurement of maximal muscle torque, total work, and work slope using only one procedure. The good reproducibility of isokinetic protocols relates to the control of speed of contraction, an important variable in transforming muscle contractions into work. Potential disadvantages of an isokinetic protocol are related to cost, availability of the testing apparatus, and the need for a technician specifically training in use of the isokinetic device. The external validity of this measurement has also been questioned, since constant angular velocity is not a physiological movement. ${ }^{50}$ Standardized position, time of day and rest intervals are recognized to affect the test-retest reliability of an isokinetic procedure. ${ }^{51}$ Thus, familiarization and strict standardization of testing procedures are crucial to providing accurate assessments of limb muscle function. ${ }^{52,53}$ Notwithstanding these considerations, an endurance isokinetic protocol using 30 maximal contractions provides reliable data, an essential feature for accurate assessment of the effects of therapeutic interventions designed to improve muscle performance.

From a clinical perspective, a minimally detectable change of $10 \%$ for total work measurements at an angular velocity of $90^{\circ}$ per second compares favorably with a corresponding value of $6 \%-12.2 \%$ for quadriceps strength measurements. ${ }^{54,55}$ Therefore, our isokinetic protocol at $90^{\circ}$ per second appears as reliable as a more widely used and accepted parameter of muscle function such as strength. The isokinetic protocol also provides reliable assessment of peak torque and fatigue index. Based on the present results, a $10 \%-15 \%$ or greater change in peak torque, total isokinetic work, and fatigue index at $90^{\circ}$ per second following a given therapeutic intervention should be considered confidently as a true treatment effect.

\section{Conclusion}

Despite a small sample size, our findings support the use of a 30-maximum repetitions isokinetic muscle testing procedure at an angular velocity of $90^{\circ}$ and $180^{\circ}$ per second in sedentary patients with moderate to severe COPD and similar body composition. Measurement of total isokinetic work using an angular velocity of $90^{\circ}$ per second was highly reliable, with a $\mathrm{MDC}_{95 \%}(\%)$ value of $10 \%$, making it a procedure of choice for evaluation of quadriceps endurance in this population. Furthermore, peak torque and fatigue index could also be assessed reliably at $90^{\circ}$ per second. Evaluation of dyspnea and leg fatigue using the modified Borg scale of perceived exertion was poorly reliable and its clinical usefulness is questionable. These results should be useful in the design and interpretation of future interventions aimed at improving muscle endurance in COPD.

\section{Acknowledgments}

FR was supported by the Canadian Respiratory Health Professionals Fellowship (Canadian Lung Association), VC is a recipient of a research training award from the Fonds de recherche du Québec-Santé, and FM holds a GlaxoSmithKline/Canadian Institutes of Health research Chair on 
COPD at Universite Laval. This work was supported by the Canadian Lung Association and by le Fonds de recherche sur les maladies respiratoires J.-D.-Bégin et P.H.-Lavoie. The authors are grateful to Eric Nadreau for his technical support during the exercise testing, and to Serge Simard for his assistance with the statistical analysis.

\section{Disclosure}

The authors report no conflicts of interest in this work.

\section{References}

1. Marquis K, Debigaré R, LeBlanc P, et al. Mid-thigh muscle crosssectional area is a better predictor of mortality than body mass index in patients with COPD. Am J Respir Crit Care Med. 2002;166: 809-813.

2. Schols AM, Broekhuizen R, Weling-Scheepers CA, Wouters EF. Body composition and mortality in chronic obstructive pulmonary disease. Am J Clin Nutr. 2005;82:53-59.

3. Swallow EB, Reyes D, Hopkinson NS, et al. Quadriceps strength predicts mortality in patients with moderate to severe chronic obstructive pulmonary disease. Thorax. 2007;62:115-120.

4. Bassett DR Jr, Howley ET. Limiting factors for maximum oxygen uptake and determinants of endurance performance. Med Sci Sports Exerc. 2000;32:70-84.

5. Van't Hul A, Harlaar J, Gosselink R, Hollander P, Postmus P, Kwakkel G. Quadriceps muscle endurance in patients with chronic obstructive pulmonary disease. Muscle Nerve. 2004;29:267-274.

6. Serres I, Gautier V, Varray AL, Préfaut CG. Impaired skeletal muscle endurance related to physical inactivity and altered lung function in COPD patients. Chest. 1998;113:900-905.

7. Coronell C, Orozco-Levi M, Mendez R, Ramirez-Sarmiento A, Galdiz JB, Gea J. Relevance of assessing quadriceps endurance in patients with COPD. Eur Respir J. 2004;24:129-136.

8. van den Borst B, Gosker HR, Koster A, et al. The influence of abdominal visceral fat on inflammatory pathways and mortality risk in obstructive lung disease. Am J Clin Nutr. 2012;96:516-526.

9. Gagnon P, Maltais F, Bouyer L, et al. Distal leg muscle function in patients with COPD. COPD. 2013;10:235-242.

10. Marquis N, Debigare R, Bouyer L, et al. Physiology of walking in patients with moderate to severe chronic obstructive pulmonary disease. Med Sci Sports Exerc. 2009;41:1540-1548.

11. Maltais F, Decramer M, Casaburi R, et al. An official American Thoracic Society/European Respiratory Society statement: update on limb muscle dysfunction in chronic obstructive pulmonary disease. Am J Respir Crit Care Med. 2014;189:e15-e62.

12. Burdett R, Vanswearingen J. Reliability of 1sokinetic muscle endurance tests. J Orthop Sports Phys Ther. 1987;8:484-488.

13. Osternig LR. Isokinetic dynamometry: implications for muscle testing and rehabilitation. Exerc Sport Sci Rev. 1986;14:45-80.

14. Pincivero DM, Lephart SM, Karunakara RA. Reliability and precision of isokinetic strength and muscular endurance for the quadriceps and hamstrings. Int J Sports Med. 1997;18:113-117.

15. Maffiuletti NA, Bizzini M, Desbrosses K, Babault N, Munzinger U. Reliability of knee extension and flexion measurements using the Con-Trex isokinetic dynamometer. Clin Physiol Funct Imaging. 2007; 27:346-353.

16. Mathur S, Makrides L, Hernandez P. Test-retest reliability of isometric and isokinetic torque in patients with chronic obstructive pulmonary disease. Physiotherapy Canada. 2004;56(02):94. Available from: http://www.physiotherapy.ca/getmedia/4f79b505-18ec-4ab6-93f2a0c75f97711c/2004-PTC-56_2-test-retest-mathur_en.pdf.aspx. Accessed April 28, 2015.

17. Walter SD, Eliasziw M, Donner A. Sample size and optimal designs for reliability studies. Stat Med. 1998;17:101-110.
18. Charter RA, Feldt LS. Meaning of reliability in terms of correct and incorrect clinical decisions: the art of decision making is still alive. J Clin Exp Neuropsychol. 2001;23:530-537.

19. Vestbo J, Hurd SS, Agusti AG, et al. Global strategy for the diagnosis, management, and prevention of chronic obstructive pulmonary disease: GOLD executive summary. Am J Respir Crit Care Med. 2013;187:347-365.

20. Voorrips LE, Ravelli AC, Dongelmans PC, Deurenberg P, Van Staveren WA. A physical activity questionnaire for the elderly. Med Sci Sports Exerc. 1991;23:974-979.

21. Pincivero DM, Gear WS, Sterner RL. Assessment of the reliability of high-intensity quadriceps femoris muscle fatigue. Med Sci Sports Exerc. 2001;33:334-338.

22. Baecke JA, Burema J, Frijters JE. A short questionnaire for the measurement of habitual physical activity in epidemiological studies. Am J Clin Nutr. 1982;36:936-942.

23. VanItallie TB, Yang MU, Heymsfield SB, Funk RC, Boileau RA. Height-normalized indices of the body's fat-free mass and fat mass: potentially useful indicators of nutritional status. Am J Clin Nutr. 1990;52: 953-959.

24. Miller MR, Hankinson J, Brusasco V, et al. Standardisation of spirometry. Eur Respir J. 2005;26:319-338.

25. Wanger J, Clausen JL, Coates A, et al. Standardisation of the measurement of lung volumes. Eur Respir J. 2005;26:511-522.

26. Macintyre N, Crapo RO, Viegi G, et al. Standardisation of the singlebreath determination of carbon monoxide uptake in the lung. Eur RespirJ. 2005;26:720-735.

27. Quanjer PH, Tammeling GJ, Cotes JE, Pedersen OF, Peslin R, Yernault JC. Lung volumes and forced ventilatory flows. Report Working Party Standardization of Lung Function Tests, European Community for Steel and Coal. Official Statement of the European Respiratory Society. Eur Respir J Suppl. 1993;16:5-40.

28. Dvir Z. Isokinetics: Muscle Testing. Interpretation and Clinical Applications. 2nd ed. Edinburgh, UK: Churchill Livingstone; 2004.

29. Borg G. Psychophysical bases of perceived exertion. Med Sci Sports Exerc. 1982;14:377-381.

30. Shrout PE, Fleiss JL. Intraclass correlations: uses in assessing rater reliability. Psychol Bull. 1979;86:420-428.

31. Beckerman H, Roebroeck ME, Lankhorst GJ, Becher JG, Bezemer PD, Verbeek AL. Smallest real difference, a link between reproducibility and responsiveness. Qual Life Res. 2001;10:571-578.

32. Bland JM, Altman DG. Statistical methods for assessing agreement between two methods of clinical measurement. Lancet. 1986;1:307-310.

33. Neder JA, Jones PW, Nery LE, Whipp BJ. Determinants of the exercise endurance capacity in patients with chronic obstructive pulmonary disease. The power-duration relationship. Am J Respir Crit Care Med. 2000; 162(2 Pt 1):497-504.

34. Malaguti C, Nery LE, Dal Corso S, et al. Scaling skeletal muscle function to mass in patients with moderate-to-severe COPD. Eur J Appl Physiol. 2006;98:482-488.

35. Montgomery LC, Douglass LW, Deuster PA. Reliability of an isokinetic test of muscle strength and endurance. J Orthop Sports Phys Ther. 1989; 10:315-322.

36. Rondelli RR, Dal Corso S, Simoes A, Malaguti C. Methods for the assessment of peripheral muscle fatigue and its energy and metabolic determinants in COPD. J Bras Pneumol. 2009;35:1125-1135.

37. Thorstensson A, Grimby G, Karlsson J. Force-velocity relations and fiber composition in human knee extensor muscles. J Appl Physiol. 1976; 40:12-16.

38. Frontera WR, DeLisa JA. Physical Medicine and Rehabilitation: Principles and Practice. 5th ed. Philadelphia, PA, USA: Wolters Kluwer/ Lippincott Williams \& Wilkins Health; 2010.

39. Butcher SJ, Meshke JM, Sheppard MS. Reductions in functional balance, coordination, and mobility measures among patients with stable chronic obstructive pulmonary disease. J Cardiopulm Rehabil. 2004;24: 274-280.

40. Lamb KL, Eston RG, Corns D. Reliability of ratings of perceived exertion during progressive treadmill exercise. Br J Sports Med. 1999;33: 336-339. 
41. Chen MJ, Fan X, Moe ST. Criterion-related validity of the Borg ratings of perceived exertion scale in healthy individuals: a meta-analysis. J Sports Sci. 2002;20:873-899.

42. Mador MJ, Rodis A, Magalang UJ. Reproducibility of Borg scale measurements of dyspnea during exercise in patients with COPD. Chest. 1995; 107:1590-1597.

43. O'Donnell DE, Travers J, Webb KA, et al. Reliability of ventilatory parameters during cycle ergometry in multicentre trials in COPD. Eur Respir J. 2009;34:866-874.

44. Frontera WR, Hughes VA, Dallal GE, Evans WJ. Reliability of isokinetic muscle strength testing in 45- to 78-year-old men and women. Arch Phys Med Rehabil. 1993;74:1181-1185.

45. Couillard A, Maltais F, Saey D, et al. Exercise-induced quadriceps oxidative stress and peripheral muscle dysfunction in patients with COPD. Am J Respir Crit Care Med. 2003;167:1664-1669.

46. Couillard A, Koechlin C, Cristol JP, Varray A, Prefaut C. Evidence of local exercise-induced systemic oxidative stress in chronic obstructive pulmonary disease patients. Eur Respir J. 2002;20:1123-1129.

47. Koechlin C, Maltais F, Saey D, et al. Hypoxaemia enhances peripheral muscle oxidative stress in chronic obstructive pulmonary disease. Thorax. 2005;60:834-841.

48. Allaire J, Maltais F, Doyon JF, et al. Peripheral muscle endurance and the oxidative profile of the quadriceps in patients with COPD. Thorax. 2004;59:673-678.
49. Janaudis-Ferreira T, Wadell K, Sundelin G, Lindstrom B. Thigh muscle strength and endurance in patients with COPD compared with healthy controls. Respir Med. 2006;100:1451-1457.

50. Mador MJ, Kufel TJ, Pineda LA, et al. Effect of pulmonary rehabilitation on quadriceps fatiguability during exercise. Am J Respir Crit Care Med. 2001;163:930-935.

51. Bottaro M, Ernesto C, Celes R, Farinatti PT, Brown LE, Oliveira RJ. Effects of age and rest interval on strength recovery. Int J Sports Med. 2010;31:22-25.

52. Selig SE, Carey MF, Menzies DG, et al. Reliability of isokinetic strength and aerobic power testing for patients with chronic heart failure. J Cardiopulm Rehabil. 2002;22:282-289.

53. Bosquet L, Maquet D, Forthomme B, Nowak N, Lehance C, Croisier JL. Effect of the lengthening of the protocol on the reliability of muscle fatigue indicators. Int J Sports Med. 2010;31:82-88.

54. Kean CO, Birmingham TB, Garland SJ, Bryant DM, Giffin JR. Minimal detectable change in quadriceps strength and voluntary muscle activation in patients with knee osteoarthritis. Arch Phys Med Rehabil. 2010; 91:1447-1451.

55. de Carvalho Froufe Andrade AC, Caserotti P, de Carvalho CM, de Azevedo Abade EA, da Eira Sampaio AJ. Reliability of concentric, eccentric and isometric knee extension and flexion when using the REV9000 isokinetic dynamometer. J Hum Kinet. 2013;37:47-53.
International Journal of COPD

\section{Publish your work in this journal}

The International Journal of COPD is an international, peer-reviewed journal of therapeutics and pharmacology focusing on concise rapid reporting of clinical studies and reviews in COPD. Special focus is given to the pathophysiological processes underlying the disease, intervention programs, patient focused education, and self management protocols.

\section{Dovepress}

This journal is indexed on PubMed Central, MedLine and CAS. The manuscript management system is completely online and includes a very quick and fair peer-review system, which is all easy to use. Visit $\mathrm{http} / / / \mathrm{www}$.dovepress.com/testimonials.php to read real quotes from published authors. 